\title{
China y la economía política de la pandemia en América Latina y el Caribe en la conturbada geopolítica del siglo XXI \\ (Ensayo)
}

\section{Javier Vadell y Florencia Rubiolo}

\author{
Javier Vadell es Doutor em Ciências Sociais, Coordenador do Programa de Pós Graduação em Relações \\ Internacionais da Pontifícia Universidade Católica de Minas Gerais, Brasil. \\ E-mail: javier.vadell@gmail.com
}

Florencia Rubiolo es Doctora en Relaciones Internacionales y Directora del Doctorado en Relaciones Internacionales de la Universidad Católica de Córdoba, Argentina.

E-mail:frubiolo@gmail.com

La pandemia del nuevo coronavirus aceleró y amplificó los procesos preexistentes en la economía política global, así como la relación tensa y cooperativa entre las grandes potencias. La crisis de la globalización neoliberal y, paralelamente, del multilateralismo; la inexistencia de un proveedor de bienes públicos globales; la inconsistencia de líderes populistas para enfrentar de manera prudente y fundamentada en términos científicos la crisis sanitaria; las tendencias proteccionistas de los Estados Unidos (EEUU); la fragilidad de los sistemas públicos de salud en contextos de creciente desigualdad social; y el irreversible ascenso de China, que derivó en una "guerra" tecnológica y comercial con Washington, son algunas expresiones de estos procesos.

En simultáneo, se observan pronósticos sombríos y de incertidumbre de cara al futuro próximo de las economías mundiales. Con excepción de China, que tuvo un crecimiento de más del $11 \%$ en el segundo trimestre de 2020, después de una caída del $10 \%$ en el primero, para el resto de los países se prevé una fuerte recesión. Para América Latina y el Caribe, la CEPAL proyecta una caída del producto interno bruto de $-9,1 \%$, lo cual la convierte en la región en desarrollo con peores pronósticos para 2020. El comercio internacional de la región también tendrá una pronunciada caída que se estima en un $23 \%$, como consecuencia de los efectos económicos derivados de la pandemia.

En este escenario, la realidad parece más compleja que una simple aceleración de procesos preexistentes. A partir de esta lectura, en la cual la pandemia es vista como un acelerador de procesos -crisis económica, liderazgo de China, entre otros-, poco tendríamos que preocuparnos por las incertidumbres; alcanza solo con lo dramático de los resultados. Sin embargo, la pandemia, como todo fenómeno en el proceso histórico, tiene dos caras: la de la continuidad y la de la ruptura.

Desde la irrupción de la pandemia de Covid-19, no existe una línea preestablecida, sino una concatenación de eventos con efectos diversos y contradictorios que afectaron no solo la vida social en diferentes lugares del mundo, sino también la configuración de fuerzas en la economía capitalista global y entre las grandes potencias. En este escenario de la economía política global, observamos tres tendencias con dinámicas diversas y contradictorias que repercuten de manera trágica 
en el mundo y, en particular, en América Latina y el Caribe: Congelamiento, aceleración y exposición de procesos sociales, económicos y políticos.

\section{Congelamiento, aceleración y exposición}

La tendencia del capitalismo se dirige hacia la acumulación renovada y constante de capital con el objetivo de acumular más capital. Cuando se quiebra esta dinámica, el sistema entra en crisis. Los obstáculos o barreras a la acumulación, expresaba Marx, deben ser superados espacial y geográficamente. Cuando el capital se enfrenta a una "barrera" en su proceso tendencial a la acumulación, en algún momento, la superará por intermedio del comercio, de inversiones en geografías distantes o vía mercado financiero transnacionalizado. El proceso de superación de obstáculos no está exento de contradicciones y conflictos sociales y políticos, ni tampoco de modificaciones de la percepción de la "realidad". La metáfora de Marx de "anulación del espacio por el tiempo" ilustra el proceso.

Con la irrupción de la pandemia, un gran obstáculo surgió, y se produjo una inversión dramática en este proceso. Lo que es dinámico por "naturaleza", de repente se congeló, se solidificó y produjo el efecto inverso: la anulación del tiempo por el espacio. Individuos confinados interactúan con recursos tecnológicos, aprovechándose de la simultaneidad y la transterritorialidad que la globalización estimuló, en palabras de Scholte. Al mismo tiempo, los gobiernos colocaron restricciones a la movilidad, reglamentaron de una u otra manera el tránsito de las personas dentro y fuera de las fronteras y movilizaron las fuerzas represivas para lidiar con la emergencia sanitaria.

Asimismo, en un primer momento, las protestas sociales y manifestaciones se paralizaron en muchos países de la región, como en los casos de Chile y Ecuador, debido a los riesgos de contagio. La parálisis fue la oportunidad para suspender o posponer elecciones en varios Estados, ya sean nacionales, provinciales o municipales. Una investigación de la CNN muestra que 65 países han decidido posponer elecciones en el año 2020, como resultado de la emergencia de la pandemia de Covid-19. En la región de América Latina y el Caribe, 10 países han postergado las elecciones en unidades subnacionales o nacionales y, en el caso de Chile, se postergó un plebiscito. En algunos casos, estas suspensiones actuaron como ollas de presión para nuevas protestas, que tuvieron que ser contenidas. El caso paradigmático fue el de Bolivia, donde finalmente se llegó a un acuerdo para que sus elecciones nacionales se realicen el 18 de octubre de este año. Las tentativas de desestabilización al régimen de Venezuela también se congelaron por el momento, inclusive por la complejidad geopolítica que llevó al presidente de los Estados Unidos, Donald Trump, en junio de 2020, a expresar que no está fuera de las posibilidades una conversación con el presidente venezolano, Nicolás Maduro. En Brasil, en un primer momento, las reformas neoliberales de ajuste y privatizaciones tuvieron que ser paralizadas.

No obstante, esto tiene vida corta. El descongelamiento es inevitable al ritmo de formas más o menos veladas de desobediencia social y de protestas reivindicativas. La pandemia se presenta como un obstáculo dramático para la expansión del 
capital. El dilema no es si el obstáculo será superado, sino cómo, en la región más desigual del planeta y donde el impacto de la pandemia fue más dramático.

Al mismo tiempo que consolidó tendencias, la pandemia desnudó las relaciones sociales y las fragilidades del orden internacional liderado por los Estados Unidos. Expuso las profundas desigualdades sociales nacionales, regionales, y las desigualdades entre los Estados, además del carácter predatorio de las élites latinoamericanas y el racismo estructural en países con fuerte pasado esclavista, entre los que se incluyen Brasil y Estados Unidos. Según la CEPAL y la Organización Internacional del Trabajo (OIT), aproximadamente el 50\% de la población de América Latina y el Caribe vive del trabajo informal, y la pandemia exhibió la fragilidad de esos grupos sociales. El coronavirus expuso las dificultades estructurales que los gobiernos, en los planos nacionales, provinciales y municipales, tuvieron para reaccionar; los sistemas públicos de salud muy precarios -o inexistentes en algunos casos-; el racismo encubierto y el desprecio a las minorías por parte de élites retrógradas. Se desvanecieron las ilusiones neoliberales de los modelos ejemplares como Chile y Perú. Perú es el país que más muertes por millón de habitantes posee. En la lista de los primeros puestos también están Chile, Bolivia y Brasil. Los cadáveres de Guayaquil fueron el primer acto de esta tragedia, mientras que los más de 130 mil muertos de Brasil continúan la obra en un escenario de desolación, donde discursos sobre la inminente vacuna sirven de consuelo.

Finalmente, y solo después de congelar y exhibir, la pandemia aceleró procesos, pero no en sentido lineal. La aceleración se da de manera arrítmica, explosiva e imprevisible en diferentes localidades, regiones y países. La crisis económica no es una excepción, como muestran las pésimas proyecciones en la retracción del PBI del CitiBank y recientemente de la OCDE.

El falso dilema entre cuidar las economías o cuidar las vidas también se desvanece en el discurso vacío en todos los rincones del planeta. Las tres "fases" de la pandemia no son secuenciales, no son etapas, sino que se manifiestan como fenómenos superpuestos y descoordinados. Las ansias por volver rápidamente a una "normalidad" pre-pandemia y pensar que el Covid-19 está controlado se muestra como una ilusión. Las demandas sociales y de sectores de clase hacia los gobiernos para volver a una normalidad colisiona con la falta de experiencia y conocimiento de los administradores públicos y de los gestores de la salud frente a un hecho inédito (la fiebre española es de inicios del siglo XX) en la mayor parte del planeta, a excepción de algunos países asiáticos que recientemente sufrieron epidemias como el SARS y diferentes tipos de gripes.

El nuevo coronavirus expuso también las fragilidades del multilateralismo, los peligros de la dependencia en la cadena global de producción y distribución de los medicamentos y materiales médicos, así como la ausencia de compromiso de los Estrados Unidos como estabilizador y proveedor de bienes públicos globales, un rol auto atribuido para el mundo Occidental después de la Segunda Guerra Mundial.

La utilización política de la pandemia se presenta como hecho natural en muchos países, como forma de canalizar la gran ansiedad y deseos de retorno a una nor- 
malidad pre-pandemia. Frente a este escenario de incertidumbre y fragmentación regional, la cooperación con China ingresó por los intersticios de las necesidades de América Latina y el Caribe.

\section{La ayuda de China y las perspectivas para la región}

Con velocidad y alcance inesperado, el coronavirus se convirtió en el foco de atención mundial de la nueva década. El coronavirus se presenta como un desafío disruptivo para el mundo hoy porque, a diferencia de crisis anteriores, la amenaza no tiene un origen financiero. A su vez, frente a la naturaleza nueva del fenómeno, tanto mercados como gobiernos deben desarrollar estrategias innovadoras para contener su avance y lidiar con las consecuencias.

A partir de la pandemia y la necesidad de respuestas inmediatas y superadoras, la dimensión sanitaria irrumpió como un nuevo eje de disputa en la competencia Washington-Beijing. Se configura de esta forma lo que denominamos una incipiente geopolítica sanitaria para América Latina y el Caribe, es decir, un reordenamiento de las prioridades y cálculos costo-beneficio en términos de capacidades, socios y herramientas, con relación a la cooperación y ayuda internacional en cuestiones sanitarias. Desde el estallido de la pandemia de Covid-19, la República Popular China se ha puesto a la vanguardia de la cooperación sanitaria en lo que se conoce como "diplomacia de los barbijos".

Esta diplomacia consiste en una política de cooperación y ayuda por parte de China hacia algunos países afectados de manera dramática por el Covid-19, que van desde el Sudeste de Asia, Irán e Italia hasta los países de América Latina y el Caribe. Se distinguen dos grandes dimensiones. La más blanda -en términos de Joseph Nye- incorpora lo discursivo, simbólico y cultural de la política de cooperación de Beijing. La segunda dimensión es la material, que se refiere a las donaciones de equipamientos y elementos médicos, envío de especialistas, etcétera.

El creciente flujo de donaciones chinas a los países afectados, y a América Latina y el Caribe en particular, ha generado intensos debates, tanto desde los objetivos ulteriores de Beijing como de las implicancias en el contexto de la competencia con Washington en términos discursivos, económicos, tecnológicos y geopolíticos. Las aproximaciones pueden ser múltiples y es aún temprano para aventurar comparaciones certeras, sumado a la dificultad de cuantificar exactamente el valor de las donaciones de cada actor. No obstante, un repaso por las acciones implementadas y su vinculación con estrategias globales permite echar luz sobre posibles escenarios futuros, a los que América Latina y el Caribe deben prestar atención sostenida en un contexto plagado de incertidumbres.

La nueva geopolítica sanitaria de China en el mundo y en la región no es un hecho coyuntural aislado. Tiene sus fundamentos en la iniciativa lanzada en 2015, conocida como la Ruta de la Seda Sanitaria (Health Silk Road), complemento de la mega iniciativa llamada "Franja económica de la Ruta de la Seda y la Ruta de la Seda marítima del Siglo XXI", conocida en inglés como Belt and Road Initiative (BRI). 
La dimensión de la salud se consideró como parte de los primeros documentos emitidos por el gobierno para pensar BRI en un sentido holístico de cooperación. Como se indica en el documento Visión y acciones de 2015:

Debemos fortalecer la cooperación con los países vecinos en el intercambio de información sobre epidemias, el intercambio de tecnologías de prevención y tratamiento y la capacitación de profesionales médicos, y mejorar nuestra capacidad para abordar conjuntamente emergencias de salud pública.

El compromiso de China con la salud global no es una tendencia nueva, sino que se remonta a la fundación de su Estado moderno. Poco después de 1949, el nuevo gobierno comenzó a participar en la asistencia sanitaria, particularmente hacia los países comunistas. Durante los años sesenta, África se convirtió en el destino de los programas de salud de China. La ayuda y los programas de China hacia el continente aumentaron, en paralelo con la profundización de los lazos diplomáticos. Más recientemente, durante la crisis del Ébola en 2012-2014, China lanzó una respuesta sin precedentes a la epidemia en África occidental, que se convirtió en su mayor ayuda de emergencia de salud en el extranjero.

Con respecto al BRI, Beijing no ha descuidado el aspecto de la salud en la iniciativa. La naturaleza flexible y adaptable del proyecto permitió introducir la idea de Health Silk Road (HSR) como una extensión de un compromiso chino con la salud global en las últimas décadas. La idea de la HSR se mencionó por primera vez en 2015, cuando la Comisión Nacional de Salud y Planificación Familiar dio a conocer el plan trienal para el intercambio y la cooperación en salud en la Franja y la Ruta (2015-2017). En enero de 2017, Xi firmó un Memorando de Entendimiento (MoU) con la Organización Mundial de la Salud que avaló la regulación sanitaria internacional y promovió la seguridad sanitaria en la Ruta de la Seda. En agosto de 2017, China organizó la Reunión de alto nivel de Belt and Road para promover la cooperación en salud, y el documento resultante fue el Comunicado de Beijing de The Belt and Road Health Cooperation \& Health Silk Road, que fue adoptado, entre otros socios, por la OMS, ONUSIDA y OCDE.

En nuestro continente, Beijing ha implementado ambas dimensiones de la diplomacia del barbijo a partir de mecanismos de cooperación sanitaria en dos planos: multilateral y bilateral. Las experiencias bilaterales van más allá de los mecanismos tradicionales Estado-Estado, sino que son de carácter multidimensional y multinivel e involucran un abanico de actores diversos. También se observa que la cooperación asume un carácter complejo: a) intergubernamental; b) entre unidades subnacionales entre sí o entre dichas unidades y los gobiernos nacionales; y c) entre grandes empresas chinas y gobiernos nacionales/subnacionales.

En el plano multilateral, debemos destacar el papel de la CELAC. Si bien esta institución, que aglutina los 33 países de América Latina y el Caribe, no es lo suficientemente fuerte en un escenario regional fragmentado, la CELAC fue la única instancia de diálogo multilateral entre funcionarios latinoamericanos y especialistas chinos. México, en su calidad de presidente pro témpore (PPT) de la CELAC 
tuvo un rol destacado en ese acercamiento. En enero, bajo liderazgo mexicano, el organismo estimuló una comunicación consensuada para el seguimiento de los brotes de coronavirus en el mundo. En marzo de 2020, la CELAC, por intermedio del canciller mexicano Ebrard, convocó una reunión ministerial virtual de Ministros de Salud de los miembros con la Comisión Nacional de Salud de China, por medio de su Vicepresidente, el doctor Zeng Yixin. Participaron 30 Ministros, además de organizaciones como la Organización Panamericana de la Salud, la CEPAL, el Caricom, el SELA, etcétera.

En el plano bilateral, la acción de China no se hizo esperar. A partir de la experiencia adquirida en el despliegue de donaciones en el Sudeste de Asia y algunos países europeos, que atravesaron los picos de la epidemia antes que nuestra región, Beijing logró movilizar recursos con antelación a cualquier otro poder internacional para asistir a América Latina. Argentina, aunque no es el mayor destino de las donaciones en la región, se convirtió en uno de los primeros receptores, con el envío de donaciones de dos cámaras térmicas por parte de Huawei en marzo. Entre los centenares de envíos realizados, en el nivel municipal desde China se destacan los de Shanghai, Hangzhou y Chongqing a Argentina, Brasil y Ecuador. Las donaciones se concentran en mascarillas, respiradores, termómetros y material médico en general. Entre las principales empresas -en su mayoría conglomerados estatales y privados chinos (SOEs) - que enviaron donaciones de envergadura a la región figuran COFCO, Huawei, Alibaba Foundation, Sinopec, BGI, Three Gorges Corporation, por mencionar algunas. Se suman a ellas los bancos estatales, como el Banco de China, ICBC y el Banco de Desarrollo de China. Además, en este complejo tejido, deben agregarse las asociaciones civiles chinas en los países latinoamericanos, como las de São Paulo y Lima, por ejemplo.

Es indiscutible que China se ha vuelto más proactiva en la gobernanza mundial de la salud desde principios de siglo. Sin embargo, sus motivaciones y objetivos, como en el caso de otras grandes potencias, van más allá de la voluntad de mejorar la salud y la seguridad humana en los países en desarrollo. El compromiso con la salud, en forma de ayuda, asistencia y cooperación, también se utiliza como una forma de poder blando que cumple con los objetivos de la política interna y externa, incluida la seguridad sanitaria, el crecimiento económico y los intereses comerciales. Los analistas internacionales estamos frente a un nuevo desafío. La diplomacia de los barbijos puede representar un anticipo de la proyección de la Ruta de la Seda Sanitaria, expresión del poder blando chino que es un efecto causal -y no casual- de la fortaleza económica de China, su poder duro. Se trata de una faceta complementaria de una diplomacia económica global que está también configurando una nueva geopolítica sanitaria en la región.

\section{Horizontes y aprendizajes para la post-pandemia}

Para América Latina y el Caribe, los efectos de la pandemia no son totalmente inesperados, ni impredecibles. Probablemente tampoco lo sean para la sociedad internacional, si pensamos que fueron los propios quiebres del desigual y poco administrado proceso de globalización los que sentaron las bases para una absolu- 
ta incapacidad de respuesta coordinada a esta catástrofe global. Sin embargo, en nuestra región, que palpamos y sentimos tan de cerca, las deudas y los desafíos a futuro nos interpelan más profundamente.

Pensar futuros escenarios y aprendizajes debe tener como centro una nueva lectura e implementación del multilateralismo. Para nuestra región, el horizonte es desalentador. La reciente disputa por la presidencia del BID, que culminó con la consagración al cargo de un candidato estadounidense por primera vez en la historia del organismo, no puede verse más que como una derrota del consenso latinoamericano y caribeño. Si la globalización alentó la disolución de fronteras vía transnacionalización, también nos puso frente al desafío de enfrentar las crisis derivadas de este proceso a escala global. La actual crisis pone a los Estados frente al desafío de encontrar respuestas innovadoras, adaptativas e integrales. Por esto, se trata de una coyuntura histórica para que el multilateralismo muestre su esencia como mecanismo de consenso y superación de problemas comunes. Será la única vía para encontrar soluciones eficaces y sostenibles a largo plazo y para que la integración, como idea y como práctica, resista.

Un segundo aprendizaje debe estar orientado a fortalecer y dar nuevo estímulo a la cooperación regional multinivel, entre actores nacionales, unidades subnacionales (Estados, provincias y municipios) y pluri-actor (empresas, organizaciones internacionales, organizaciones no gubernamentales y de la sociedad civil). Esta perspectiva se encuentra en línea con lo planteado por la Agenda 2030 y la centralidad dada a las alianzas multiactor como canales necesarios para la consecución de los ODS. Los complejos entramados de vínculos entre diferentes agencias, instituciones, organizaciones y personas que atraviesan las fronteras estatales fortalecen la capacidad de toma de decisiones y de respuesta regional. Estos lazos, ya establecidos y muchos de ellos consolidados, pueden funcionar como plataformas para una mayor integración en América Latina y el Caribe, a pesar de las diferencias que prevalezcan en el nivel de los ejecutivos nacionales.

Directamente relacionado con la dimensión sanitaria, la pandemia impactó en la región con mayor fuerza debido a la fragmentación de los sistemas de salud, la protección social y las brechas estructurales históricas. Los países de la región implementaron medidas tímidas, poco articuladas y selectivas, que reflejan la ausencia de solidaridad y la falta de liderazgo regional ante una crisis con escasos precedentes. Se viene planteando la impostergable necesidad de promover una mayor cooperación sanitaria extra regional que involucre a la OMS, a China, a los Estados Unidos, a la UE, a Rusia, entre otros actores, ante las consecuencias profundas que la pandemia dejará a nivel humano y ambiental en nuestra región.

Finalmente, la recuperación post-pandemia no será inmediata. En este sentido, el estímulo a la construcción de infraestructura para fortalecer un desarrollo sustentable y sostenido, tanto nacional como regional, con foco en la interconectividad, la tecnología y las comunicaciones también deberá ser prioritario. El rol de China puede ser neurálgico.

En cuanto al impacto en el desarrollo sustentable de los países de América Latina y el Caribe, la BRI puede jugar un papel clave en el futuro energético, la 
reconversión de la matriz de energía y la mitigación del cambio climático. Aunque el vertiginoso crecimiento de China ha tenido altos costos en materia ambiental y aumento de desigualdades entre zonas rurales y urbanas, la orientación de su último Plan quinquenal hace foco en la necesidad de reducir la utilización de carbón y la emisión de gases de efecto invernadero, en línea con el compromiso asumido en 2016 en el Acuerdo de París.

América Latina y el Caribe pueden encontrar un socio sin precedentes para una estrategia de largo plazo de desarrollo inclusivo a través del financiamiento de China. No obstante, hay dilemas que deberán enfrentar los gobiernos desde una perspectiva multilateral. Los acuerdos de acceso a la Iniciativa son de naturaliza bilateral, lo cual genera escasos incentivos a la búsqueda de consensos regionales para articular los vínculos con el gigante asiático. De esta manera, se observa que, dentro de los bloques o procesos de integración latinoamericanos, algunos países han ingresado a la BRI y otros no. Tanto en la Alianza del Pacífico como en el Mercosur, se encuentran divididas las aguas al respecto. Es fundamental que los Estados logren alcanzar cierto grado de consenso en las negociaciones de los fondos y la regulación de los proyectos -entre otros puntos- para que la región logre potenciar los beneficios de una mayor presencia china, y evitar que profundice las asimetrías ya existentes en América Latina y el Caribe. Este horizonte, a la luz de la actual situación de la integración en América Latina y el Caribe, parece una utopía. No obstante, resignarse a una nueva inserción basada en la división y la competencia intrarregional perpetuará las condiciones de dependencia y desigualdad que la región necesita superar para encontrar un camino genuino de desarrollo.

Javier Vadell y Florencia Rubiolo, "China y la economía política de la pandemia en América Latina y el Caribe en la conturbada geopolítica del siglo XXI (Ensayo)". Revista Temas y Debates. ISSN 1666-0714, año 24, número especial, julio-diciembre 2020, pp. 43-50. 